$10 \mid 2021$

Intelligence artificielle, pratiques sociales et politiques publiques

\title{
La collaboration sur les plateformes d'innovation sociale : le cas « Solidarité Covid-19 Francophonie »
}

Collaboration on social innovation platforms : the "Solidarité Covid-19

Francophonie "case

Colaboración en plataformas de innovación social : el caso "Covid-19

Solidaridad"

\section{Damien Renard}

\section{OpenEdition}

Journals

Édition électronique

URL : https://journals.openedition.org/ctd/5035

DOI : $10.4000 /$ ctd. 5035

ISSN : 2491-1437

Éditeur

Chaire Unesco Pratiques émergentes en technologies et communication pour le développement

Édition imprimée

ISBN : 2491-1437

Référence électronique

Damien Renard, «La collaboration sur les plateformes d'innovation sociale : le cas « Solidarité Covid-19 Francophonie » », Communication, technologies et développement [En ligne], 10 | 2021, mis en ligne le 20 mai 2021, consulté le 27 mai 2021. URL : http://journals.openedition.org/ctd/5035 ; DOI : https://doi.org/10.4000/ctd.5035

Ce document a été généré automatiquement le 27 mai 2021.

Communication, technologies et développement 


\title{
La collaboration sur les plateformes d'innovation sociale : le cas «Solidarité Covid-19 Francophonie »
}

\author{
Collaboration on social innovation platforms : the " Solidarité Covid-19 \\ Francophonie "case \\ Colaboración en plataformas de innovación social : el caso "Covid-19 \\ Solidaridad"
}

Damien Renard

Les plateformes sont des infrastructures permettant à des groupes d'acteurs distincts d'œuvrer de concert (Prpić et al., 2015 ; Renard \& Davis, 2019). Leur trait principal est donc leur capacité à coordonner des «myriades de micro-activités " (Benavent, 2016, p. 33). Dans le domaine de l'innovation sociale, le mouvement de plateformisation que traversent nos sociétés constitue une opportunité. Par exemple, durant la pandémie Covid-19, on a observé l'émergence de plusieurs collectifs ayant pour objectif d'apporter une solution aux problèmes engendrés. Sur Twitter, le mouvement «\#PourEux » s'est donné pour mission de subvenir aux besoins essentiels des sansabris. Sur Slack, la "communauté Solidarité-Covid-19 Francophonie ", soutenue par l'Organisation Internationale de la Francophonie, a rassemblé des amateurs et des experts du monde francophone. Si ces collectifs constituent une source d'innovations sociales, ils ne peuvent fonctionner sans un réseau de personnes suffisamment engagées dans une activité collaborative. La collaboration renvoie à l'idée de travailler ensemble en encourageant le dépassement de situations où le travail individuel n'est pas suffisant pour obtenir les ambitions désirées (Huxham, 1996). Dès lors, un des enjeux est de réussir à proposer des expériences capables d'engager les participants dans un processus collaboratif qui puisse favoriser la production d'innovations sociales. 
2 Pour ce faire, les concepteurs des plateformes travaillent sur la mise en place de dispositifs socio-techniques dont l'objectif est précisément de stimuler les interactions, que ce soit entre les utilisateurs et/ou avec la plateforme. Les objets socio-techniques relevant de l'intelligence artificielle (IA) s'inscrivent dans cette perspective en développant des systèmes communicationnels intelligents. En effet, de par sa capacité à agir avant, pendant et après l'action des utilisateurs (Kreutzer \& Sirrenberg, 2020), l'IA est susceptible de jouer un rôle fondamental dans le processus collaboratif. Dans le cadre de cet article, nous nous sommes intéressés aux formes que revêt l'expérience collaborative et au rôle de l'IA dans ce processus. Nous avons étudié la communauté Solidarité Covid-19 Francophonie ${ }^{1}$ lancée dans le cadre de la crise sanitaire sur la plateforme collaborative Slack. Nous y avons mené une enquête ethnographique durant deux mois, et complété ce travail d'observation par une analyse quantitative. Les résultats mettent en lumière un degré élevé d'hétérogénéité entre les acteurs, que ce soit au niveau du secteur d'activité ou du pays d'appartenance. Dans ce cadre, les technologies d'IA, au même titre que les animateurs de communautés, participent à définir le système d'interactions entre les acteurs en facilitant la circulation de ressources affectives et cognitives. De manière paradoxale, notre analyse révèle la difficulté de la plateforme à inclure des publics plus éloignés alors même que l'innovation sociale a pour mission d'apporter des réponses aux défis sociétaux. Dans la première partie de cette recherche, plusieurs concepts issus de la littérature sont exposés. La seconde partie présente les résultats de l'étude mixte. Dans la dernière partie, nous concluons sur les limites et les futures pistes de recherche suscitées par nos travaux.

\section{Revue de littérature}

\section{L'innovation sociale}

3 L'innovation sociale constitue par essence le moyen d'offrir de nouvelles solutions capables de résoudre plus efficacement les problèmes et les besoins sociaux et de favoriser le mieux-être des individus. Autrement dit, elle vise à offrir des réponses nouvelles à des besoins sociaux non satisfaits dans les conditions actuelles du marché et des politiques sociales. L'innovation sociale est par définition un objet protéiforme. Elle peut désigner aussi bien un produit ou service qu'un processus (ex.mode d'organisation, de distribution) dans des domaines comme le vieillissement, la petite enfance, le logement, la santé, la lutte contre la pauvreté, l'exclusion. Selon la littérature, les innovations sociales partagent trois caractéristiques (Lee et al. , 2019) :

1. Le développement d'une solution dans un contexte donné: les innovations sociales s'insèrent dans un contexte spécifique et visent à répondre à un problème social spécifique.

2. Un périmètre local : les innovations sociales s'insèrent dans des marchés géographiquement limités, ce qui rend leur généralisation difficile.

3. L'hétérogénéité des domaines: la notion d'innovation sociale implique une variété de domaines (ex. : médecine, environnement, etc.) et de disciplines.

4 Comme le rappelle Prades (2015), les innovations sociales sont par définition accrochées à un territoire naturel, culturel, juridique ou économique. Aussi, se pose la question de la standardisation et de la circulation de ces innovations entre des acteurs ancrés dans des mondes pluriels. Dans cette perspective, il y a une véritable 
interrogation sur les possibilités de faire collaborer des publics hétérogènes, ancrés dans des cultures locales, mais capables aujourd'hui d'interagir via les technologies de l'information et de la communication.

\section{Les plateformes et les collectifs d'acteurs}

Les plateformes sont des infrastructures permettant à des groupes d'acteurs distincts d'œuvrer de concert. L'expansion de pratiques et de dispositifs dits participatifs en ligne représente une opportunité pour les organisations publiques et privées. Pour les organisateurs, elles sont « un moyen d'obtenir des gains de productivité inégalés en exploitant les actifs sous-utilisés et en générant par l'agrégation des informations un apprentissage exponentiel des comportements et des habitudes" (Benavent, 2016, p. 21). Dans le domaine de l'innovation sociale, les plateformes permettent de mobiliser des porteurs de projets et/ou des collectifs d'acteurs dans la réalisation de projets individuels ou collectifs. Cette distinction entre l'innovation sociale produite par des individus et l'innovation sociale produite par les collectifs est importante puisqu'elle implique de penser le processus d'innovation sociale dans une perspective différente. La vision centrée sur l'individu s'inscrit dans la définition schumpetérienne de l'innovation avec une place centrale accordée à la figure de l'entrepreneur. Autrement dit, l'entrepreneur est perçu comme un agent de l'innovation. Dans cette perspective, l'innovation sociale fait intervenir la figure de l'entrepreneur social qui possèderait les traits particuliers, le caractère, l'ambition, les ressources, le courage et la persistance pour mener un projet depuis sa phase d'idéation à celle de l'action (Mumford 2003; Martin et Osberg, 2007). À côté de la figure de l'entrepreneur social, Harisson (2012) rappelle l'existence d'une autre figure centrale de l'innovation sociale, à savoir les collectivités. Dans cette vision de l'innovation sociale, les acteurs ne sont plus seulement les porteurs d'un projet individuel. Il s'agit de penser l'innovation sociale comme un processus capable de mobiliser une variété d'acteurs qui vont se coordonner pour développer des solutions visant la résolution de problèmes socio-économiques. Ces collectifs s'inscrivent dans cette évolution des pratiques dans le champ de l'innovation qui a été marqué par le développement des technologies de l'information et de la communication et la montée en puissance du paradigme de l'innovation ouverte. Néanmoins se pose la question de la coordination, le fort degré d'hétérogénéité entre les acteurs pouvant constituer un frein au processus d'innovation sociale.

\section{L'IA et l'expérience collaborative}

Dans le contexte de ces plateformes, l'IA est susceptible de jouer un rôle fondamental de par sa capacité à agir avant, pendant et après l'action des utilisateurs (Haenlein \& Kaplan, 2019). Selon Vayre (2019), l'IA est en premier lieu une discipline scientifique ayant pour objectif la compréhension des dimensions de l'intelligence humaine, et ce, afin d'en permettre la simulation par une machine. La littérature distingue généralement deux courants principaux : la branche symbolique consiste à formaliser et appliquer un raisonnement en fonction de règles logiques explicites alors que la branche connexionniste regroupe l'ensemble des techniques d'apprentissage à partir de données (apprentissage automatique, deep learning, réseaux de neurones) (Cardon et al., 2018). Ces dernières années, l'augmentation du volume de données produites par 
l'industrie numérique a conduit à un regain d'intérêt pour le paradigme connexionniste permettant le développement de systèmes capables «d'interpréter correctement des données externes, à tirer des enseignements de ces données et à utiliser ces enseignements pour atteindre des objectifs et des tâches spécifiques grâce à une adaptation souple» (Haenlein et Kaplan, 2019, p. 15) ${ }^{2}$. Au final, on assiste au développement de systèmes communicationnels intelligents capables d'offrir aux utilisateurs des expériences personnalisées, grâce au déploiement d'objets sociotechniques ayant la capacité de réaliser des tâches précises, de résoudre certains problèmes, ou encore de trouver des solutions de façon autonome. Par exemple, le recours aux «bots intelligents » permet de stimuler la présence sociale qui peut être définie comme la manière dont des individus, interagissant via un moyen technologique, ressentent qu'ils sont ensemble (Gefen et Straub, 2003 ; Shen et Khalifa, 2008). Dans le cadre de cette recherche, notre objectif est de comprendre le rôle ces objets techniques dans le processus collaboratif au sein de ces plateformes caractérisées par un degré d'hétérogénéité élevé.

\section{Méthodologie}

\section{La communauté Solidarité Covid-19 Francophonie}

7 Nous nous sommes intéressés aux formes que revêt l'expérience collaborative et au rôle de l'IA dans ce processus. Nous avons étudié le collectif Solidarité Covid-19 Francophonie ${ }^{3}$ lancé dans le cadre de la crise sanitaire et qui rassemble 1455 utilisateurs issus principalement d'une dizaine de pays francophones (Congo, Bénin, France, Belgique, etc.). Porté par l'Organisation Internationale de la Francophonie, son objectif est «de fédérer, de partager et de coconstruire des solutions innovantes, créatives pour atténuer les conséquences de la pandémie de la maladie COVID-19». Pour accompagner son développement, l'institution a choisi la plateforme de communication collaborative slack qui a pour but de faciliter la collaboration en regroupant tous les flux de communication dans un même espace (messagerie instantanée, service de partage de fichiers et plateforme de chat vidéo). La plateforme fait par ailleurs appel aux technologies d'IA dites «faibles » afin d'optimiser l'expérience utilisateur ${ }^{4}$.

Enfin, si le collectif a pour mission première d'encourager le partage des idées créatives et de projets innovants au sein de l'espace francophone, elle est également un instrument de visibilité pour les porteurs de projets. Associé à la plateforme, un site web permet en effet à chacun de déposer un ou plusieurs projets qui sont évalués par un comité d'experts mandatés par l'OIF et valorisés par l'organisation dans et hors de la plateforme. A l'issue de la période d'observation, 184 projets pour lutter contre la crise ont été répertoriés.

\section{Le choix des méthodes mixtes}

Pour analyser le processus collaboratif, nous avons privilégié les méthodes mixtes qui combinent des données quantitatives et qualitatives et qui permettent d'étudier à la fois les patterns d'usage et leur sens du point de vue des acteurs (Hesse-Biber et Griffin, 2013). La première étape a consisté à mener une enquête ethnographique sous la forme d'une observation de l'activité des usagers, l'université étant partenaire du projet ${ }^{5}$ 
(Kozinets, 2010). A l'issue de ce travail exploratoire durant 60 jours, nous avons mené une analyse quantitative de la plateforme. En faisant le choix d'un design de complémentarité (Creswell, 2017), notre objectif était de prendre en considération différents niveaux d'analyse. Nous avons étudié le profil socio-démographique des participants ainsi que leur position dans le réseau de relations (au sein de la plateforme). Pour ce faire, nous nous sommes appuyés sur la méthode d'analyse des réseaux sociaux qui repose sur des visualisations graphiques issues d'algorithmes permettant de calculer des degrés de proximité, d'importance ou de densité entre les différents acteurs d'un réseau.

\section{Résultats}

\section{La collaboration et les enjeux d'hétérogénéité}

10 L'analyse du profil des participants révèle une forte hétérogénéité entre les membres, que ce soit au niveau des compétences, du pays d'appartenance ou encore du projet supporté. Ainsi, parmi les pays les plus représentés, on observe que $19,5 \%$ des inscrits viennent du Congo, $10 \%$ du Cameroun ou encore $10 \%$ de France par exemple. Cette diversité se reflète également dans les secteurs d'activités des participants (audiovisuel, santé, commerce et d'artisanat, etc.) et dans les objectifs poursuivis, à savoir la découverte de projets et initiatives locales, la veille informationnelle, le réseautage ou encore le développement de projets :

«Bonjour! Je coordonne une association d'action sociale et nous avons pour projet de confectionner des masques de protection en tissu (AFNOR). Et nous cherchons des subventions».

«Découvrez RIIPOST, Une application mobile développée au \#Togo pour contribuer à la riposte au nouveau Coronavirus "COVID-19 ».

11 Dans la littérature, la diversité est généralement considérée comme un facteur favorable à l'innovation (McEvily et Zaheer, 1999; Nieto et Santamaría, 2007). Néanmoins, cela peut impliquer un travail important de coordination (Bell \& Zaheer, 2007 ; Rchika et al., 2021). Dès lors, le principal enjeu est de réussir à développer les échanges entre les participants en développant un système capable de modaliser et créer du lien entre les participants (Gardère et al., 2019).

\section{Une plateforme en recomposition autour des canaux thématiques}

Le dispositif participatif est articulé autour d'un objectif commun: le partage d'innovations dans l'espace francophone au service de la lutte contre les effets de la pandémie Covid-19. Au-delà de la mise en place d'un objet capable de faire lien entre les participants, la plateforme de communication collaborative a été divisée en onze canaux incluant deux généralistes et neuf canaux thématiques. Chacun de ces espaces vise à rassembler des personnes partageant un même intérêt (exemple : le tourisme, les médias, etc.). Il s'agit ainsi de favoriser le développement d'univers de discours (Clarke et Star, 2008), et de faire de chacun de ces lieux thématisés une arène à l'intérieur de laquelle les frontières soient délimitées par le sentiment de « faire ensemble ». Au final, les participants peuvent s'inscrire dans un ou plusieurs canaux qui serviront de lieux d'échange d'idées, de débats ou de présentations des projets. La plateforme devient ainsi un espace de collaboration, ouverte à tous, permettant à chacun de prendre part à 
la lutte contre la pandémie. On observe ainsi le développement d'une dynamique collaborative et contributive, que ce soit à travers la mise à disposition de ressources, de savoir-faire mais également par un travail de diffusion des connaissances acquises à un niveau plus local :

«Un chatbot sur Whatsapp développé au Nigeria pour apprendre plus sur le COVID19

Bonjour Le bot peut-il envoyer des audios de sensibilisation?

Je pense que c'est possible, c'est une intégration mais généralement les bots ne s'arrêtent qu'au texte

Magnifique, est ce que tu as accès aux développeurs de ce bot?

Oui oui, j'ai contacté le développeur, il m'a confirmé que c'est possible d'envoyer des notes vocales

Great si ça l'intéresse de partager ses connaissances, invite le à rejoindre le slack :)

Bonsoir c'est un excellent projet, j'ai eu la même idée au Bénin pour informer les populations reculées »

\section{Le rôle de l'IA dans le processus collaboratif}

13 La collaboration amène donc à questionner la structure sous-jacente mais également le rôle de la plateforme dans ce processus en tant qu'entité organisante. L'enquête ethnographique révèle l'existence d'une animation à deux niveaux. Le premier niveau est opéré par les animateurs de communauté issus de différents pays francophones. Ils exercent la fonction de "passeurs de frontières" (Aldrich et Herker, 1977) et participent à créer un climat de confiance sur la plateforme. Le second niveau est celui des objets socio-techniques qui interprètent les comportements et participent à la dynamique collaborative. Dans le contexte de la plateforme étudiée, nous nous intéressons plus particulièrement aux objets relevant de l'IA. La plateforme a intégré un premier type d'IA (système de recommandation) visant à capter l'attention par la mise en avant d'un contenu pertinent. Dans le cas présent, les utilisateurs peuvent ainsi paramétrer la plateforme et recevoir des notifications personnalisées afin de mieux gérer les flux de messages (par exemple selon des mots-clés ou des centres d'intérêts). Les objets socio-techniques facilitent ici les échanges entre des personnes similaires et/ ou complémentaires. Le second type d'IA est le recours aux agents conversationnels intelligents : le slackbot est un agent conversationnel dont une des fonctionnalités est d'apporter des réponses aux utilisateurs. Il permet d'automatiser certaines réponses et d'humaniser la plateforme même si celle-ci se caractérise par un faible niveau d'activité. Ainsi, en humanisant l'interface et en stimulant le sentiment de présence sociale, les technologies d'intelligence artificielle participent au développement d'un environnement suffisamment engageant pour stimuler la collaboration.

\section{Un collectif francophone « en construction »}

14 On observe que la lutte contre la pandémie covid-19 agrège un ensemble d'acteurs francophones autour d'un thème qui fait frontière. Ils «font collectifs » au sein d'une plateforme s'appuyant sur une série d'acteurs humains et non-humains chargés de coordonner les participants qui s'assemblent et se désassemblent. Dans cette perspective, les IAs structurent les pratiques en accompagnant de manière visible et invisible les participants. Ce processus d'accompagnement permet de créer les conditions d'un contexte expérientiel ayant pour but de favoriser de nouvelles formes 
d'interdépendances sociales (Renard et Davis, 2019). L'exemple le plus probant est celui des porteurs de projets dont l'activité sociale est orientée vers les autres alors même que les projets renvoient à des problématiques locales ou s'inscrivent dans des domaines différents. Ainsi, comme le montre la figure ci-dessous, ils sont engagés dans une dynamique collaborative avec les autres porteurs (les nœuds représentent les porteurs de projets et la couleur leur pays de résidence). La visualisation du sousréseau montre d'un côté, la forte représentation de trois pays (Congo (vert), Cameroun (bleu) et France (violet)) et de l'autre côté, l'intensité des échanges entre des acteurs issus de différents pays. Ainsi, malgré un degré d'hétérogénéité élevé, ces personnes sont engagées dans un processus d'échange encouragé par la présence d'une structure technologique souple et flexible qui participe à définir le système d'interactions entre les acteurs.

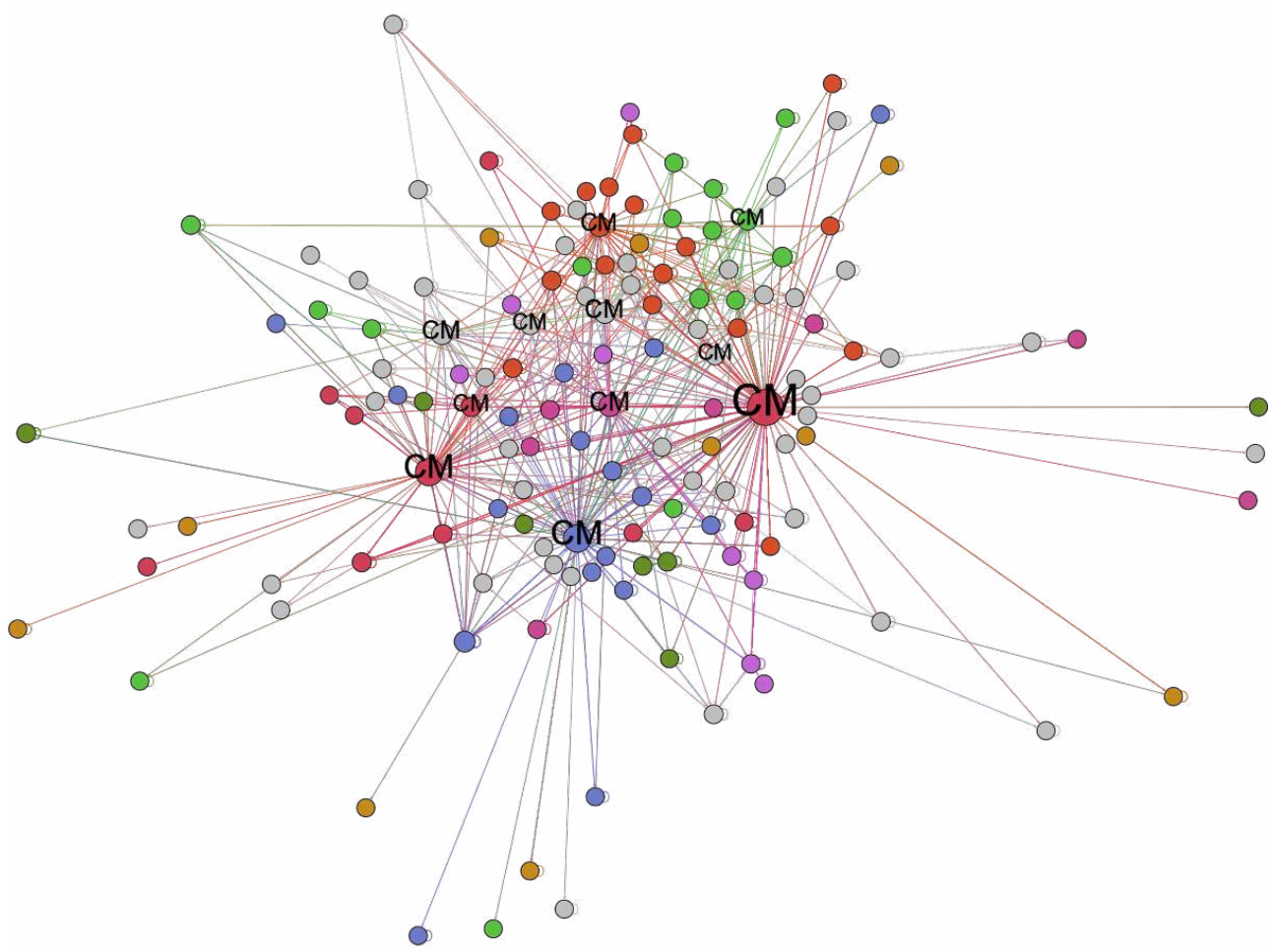

Figure. Visualisation des liens entre les porteurs de projets

\section{Discussion}

Dans le champ de l'innovation sociale, les plateformes sont des espaces clés permettant de supporter une culture collaborative en vue de favoriser le processus d'innovation, à savoir l'émergence d'idées nouvelles (Mulgan, 2006). À l'intérieur, la collaboration entre les acteurs est un enjeu majeur nécessitant de faire dialoguer des personnes poursuivant des objectifs généralement ancrés dans des problématiques locales. Les résultats de notre analyse montrent que plusieurs pratiques collaboratives co-existent : création de contenu, enrichissement de contenu (ex. soutiens ou commentaires), ou curation de contenu. On observe que le collectif en ligne peut s'appuyer sur une plateforme intégrant des technologies d'intelligence artificielle renforçant l'automatisation de certaines tâches. Ces dernières permettent la construction d'un environnement intelligent capable de construire de nouvelles formes 
d'interdépendances sociales. Dans le cas présent, l'IA joue un rôle central de par sa capacité à mettre à disposition des ressources cognitives ou affectives, transformant ces technologies en "passeurs de frontières " à côté des animateurs de communautés. Autrement dit, elle vient appuyer un collectif en train de se faire.

Néanmoins, on peut s'interroger sur la capacité de ces outils à inclure des publics plus diversifiés. S'il existe une forte hétérogénéité liée à l'ancrage culturel ou le secteur d'activité, l'analyse de la structure sociale met en lumière une certaine forme d'homogénéité parmi les participants les plus centraux dans le réseau de relations. Ainsi, au sein de la composante connexe principale (à savoir l'ensemble des acteurs reliés deux à deux par un chemin, soit un total de 427 participants), plus de $40 \%$ des personnes ont déclaré une profession/titre inscrite dans la catégorie «cadres et professions intellectuelles supérieures » et presque $70 \%$ sont des hommes. On note ainsi une importante limite de ces dispositifs technologiques qui échouent à inclure un public plus hétérogène. Or, la non-inclusion des publics plus éloignés amène plusieurs difficultés. En premier lieu, en ne parvenant pas à les intégrer, les plateformes risquent de favoriser l'entre-soi et de créer des bulles de filtre (Pariser, 2011) valorisant des projets déconnectés de la réalité et des besoins des populations visées. En second lieu, la sous-représentation de certains publics peut engendrer des biais algorithmiques. Comme le rappellent Cardon et al. (2018), «l'horizon du calcul n'appartient pas au calculateur, mais au monde qui lui a donné des exemples «étiquetés » (p. 214). Dans cette perspective, il est impératif de veiller à un équilibre de la représentation des publics dans les politiques d'innovations sociales, l'efficacité des technologies d'intelligence artificielle étant dépendante des données produites par les utilisateurs eux-mêmes.

Si cette recherche permet de mieux comprendre le rôle de l'IA comme levier de l'innovation, celle-ci présente certaines limites. En premier, nous nous sommes intéressés à une seule plateforme d'innovation sociale. Il serait intéressant de répliquer ce travail en étudiant d'autres types de collectifs évoluant sur d'autres plateformes. Par ailleurs, notre recherche révèle que le collectif se caractérise par un certain degré d'expertise et une maîtrise des outils technologiques. Aussi, se pose la question de l'acceptation et de l'adoption de ce type de technologie par une population moins experte.

\section{BIBLIOGRAPHIE}

Aldrich, H. and Herker, D. (1977). Boundary spanning roles and organization structure. Academy of Management Review, 2, 217-230. https://doi.org/10.5465/amr.1977.4409044

Bell, G., and Zaheer, A. (2007). Geography, networks, and knowledge flow. Organization Science, 18(6), 955-972. https://doi.org/10.1287/orsc.1070.0308

Benavent C. (2016). Plateformes : Sites collaboratifs, marketplaces, réseaux sociaux... Comment ils influencent nos choix. Limoges : FYP Éditions. 
Cardon, D., Cointet, J. et Mazières, A. (2018). La revanche des neurones : L'invention des machines inductives et la controverse de l'intelligence artificielle. Réseaux, 5(5), 173-220. https://doi.org/ $10.3917 /$ res.211.0173

Clarke, A. E., and Star, S. L. (2008). The Social Worlds/Arenas/Discourse Framework as a TheoryMethods Package, 113-137. In : Edward Hackett, O. ; Lynch A. M., and Wacjman J. (Eds.) Handbook of Science and Technology Studies. Cambridge, MA : MIT Press.

Creswell, J. W. (2017). Research design : qualitative, quantitative, and mixed methods approaches, $\left(4^{\text {th }}\right.$ ed), Thousand Oaks, California : SAGE Publications.

Gardere, E., Bouillon, J-L., Loneux, C. (2019). Le « collaboratif » dans les organisations : une question de communication. Communication \& Organisation, 55, 9-22. https://doi.org/10.4000/ communicationorganisation. 7650

Gefen D. and Straub, D.W. (2004). Consumer trust in B2C e-Commerce and the importance of social presence : experiments in e-products and e-services. Omega, 32(6), 407-424. http:// dx.doi.org/10.1016/j.omega.2004.01.006

Haenlein, M., and Kaplan, A. (2019). A brief history of artificial intelligence : on the past, present, and future of artificial intelligence. California Management Review, 61(4), 5-14. https://doi.org/ $10.1177 / 0008125619864925$

Harrisson, D. (2012). Social Innovation : What Is Coming Apart and What Is Being Rebuilt ?, 73-82. In H.W. Franz, J. Hochgerner \& J. Howaldt (Eds.). Challenge Social Innovation. Potentials for Business, Social Entrepreneurship, Welfare and Civil Society. Berlin, New York : Springer

Hesse-Biber, S. and Griffin, A. (2013). Internet-mediated technologies and mixed methods research : Problems and prospects. Journal of Mixed Methods Research, 7(1), 43-61. https://doi.org/ $10.1177 / 1558689812451791$

Huxham, C. (1996). Creating Collaborative Advantage. London : SAGE.

Kozinets, R. (2010). Netnography : Doing ethnographic research online. London, England : Sage.

Kreutzer, R. T., \& Sirrenberg, M. (2020). What is Artificial intelligence and how to exploit it? (pp. 1-57). In : Understanding Artificial intelligence. Management for professionals. Cham : Springer. .

Lee, R., Spanjol, J., \& Sun, S. L. (2019). Social Innovation in an interconnected world : Introduction to the special issue, Journal of Product Innovation Management, 36(6), 662-670. https://doi.org/ $10.1111 /$ jpim.12513

Libai B., Bart Y., Gensler, S., Hofacker, C., Kaplan A., Kötterheinrich K, et Kroll, E. (2020). Brave new world? On AI and the management of customer relationships. Journal of Interactive Marketing, 51, 44-56. https://doi.org/10.1016/j.intmar.2020.04.002

Martin, R. L., and Osberg, S. (2007). Social Entrepreneurship : the case for definition, Stanford Social Innovation Review, 29-39.

McEvily, B., et Zaheer, A. (1999). Bridging ties : a source of firm heterogeneity in competitive capabilities, Strategic Management Journal, 20(12), 1133-1156. https://doi.org/10.1002/ (SICI)1097-0266(199912)20:12<1133 : :AID-SMJ74>3.0.CO ;2-7

Mulgan, G. (2006). The Process of Social Innovation. Innovations : Technology, Governance, 1(2), 145162. https://doi.org/10.1162/itgg.2006.1.2.145

Mumford, M. (2003). Where have we been, where are we going ? taking stock in creativity research. Creativity Research Journal, 15(2), 107-120. https://doi.org/

10.1080/10400419.2003.9651403 
Nieto, M.J. et Santamara, L. (2007). The importance of diverse collaborative networks for the novelty of product innovation. Technovation, 27, 367-377. https://doi.org/10.1016/j.technovation. 2006.10.001

Pariser, E. (2011), The Filter Bubble: What the internet is hiding from you. New York : Penguin Press

Prades, J. (2015). Du concept d'« innovation sociale ». Revue internationale de l'économie sociale, 338, 103-112. https://doi.org/10.7202/1033876ar

Prpić, J., Shukla, P. P., Kietzmann, J. H., \& McCarthy, I.P. (2015). How to work a crowd : developing crowd capital through crowdsourcing. Business Horizons, 58(1), 77-85. https://doi.org/10.1016/ j.bushor.2014.09.005

Renard, D. et Davis, J. (2019). Social interdependence on crowdsourcing platforms. Journal of Business Research, 103, 186-194. https://doi.org/10.1016/j.jbusres.2019.06.033.

Rchika, T., Renard D., et Lambotte, F. (2021). Entre hétérogénéité : Une discussion empirique du rôle des objets-frontières dans le réseau d'innovation Ouishare. Approches Théoriques en Information et Communication, 1(2), 23 - 45.

Shen, K. N, and Khalifa, M. (2008). Exploring multidimensional conceptualization of social presence in the context of online communities. International Journal of Human-Computer Interaction, 24(7), 722-748. https://doi.org/10.1080/10447310802335789

Vayre, J.S (2019). L'intelligence des machines et l'esprit du capitalisme . Communication, 36(1). https://journals.openedition.org/communication/9726

\section{NOTES}

\section{1. https://solidaritecovid19.francophonie.org/}

2. "a system's ability to correctly interpret external data, to learn from such data, and to use those learnings to achieve specific goals and tasks through flexible adaptation"

3. https://solidaritecovid19.francophonie.org/

4. https://www.technologyreview.com/2018/01/16/104835/slack-hopes-its-ai-will-keep-youfrom-hating-slack/

5. https://uclouvain.be/fr/instituts-recherche/ilc/ilc-covid-19.html

\section{RÉSUMÉS}

Cette recherche vise à comprendre le processus de coproduction d'innovations sociales dans le contexte des plateformes numériques. À travers l'étude de la plateforme "Solidarité Covid-19 Francophonie", nous analysons comment l'intelligence artificielle facilite la collaboration entre des acteurs de cultures différentes et/ou développant des projets dans des domaines variés. Les résultats de l'étude ethnographique, complétée par une analyse quantitative, révèlent le développement des pratiques collaboratives grâce aux différents types de connecteurs, humains ou non-humains, qui organisent les échanges et facilitent la circulation de ressources affectives 
et cognitives. De manière paradoxale, l'analyse montre la difficulté de la plateforme à inclure certains publics.

This research aims to understand the process of co-production of social innovations in the context of digital platforms. Through the study of the "Solidarité Covid-19 Francophonie" platform, we investigate how artificial intelligence facilitates collaboration between actors from different cultures and/or developing projects in various fields. The results of the ethnographic study, completed by a quantitative analysis, reveal the development of collaborative practices thanks to the different types of connectors, human or non-human, which organize exchanges and facilitate the circulation of affective and cognitive resources. Paradoxically, the analysis shows the platform's difficulty in including certain audiences.

Esta investigación pretende comprender el proceso de coproducción de innovaciones sociales en el contexto de las plataformas digitales. A través del estudio de la plataforma "Solidarité Covid-19 Francophonie", investigamos cómo la inteligencia artificial facilita la colaboración entre actores de diferentes culturas y/o el desarrollo de proyectos en diversos campos. Los resultados del estudio etnográfico, completados por un análisis cuantitativo, revelan el desarrollo de prácticas de colaboración gracias a los diferentes tipos de conectores, humanos o no, que organizan los intercambios y facilitan la circulación de recursos afectivos y cognitivos. Paradójicamente, el análisis muestra la dificultad de la plataforma para incluir a ciertos públicos.

\section{INDEX}

Palabras claves : colaboración, plataforma, inteligencia artificial, innovación social

Keywords : collaboration, platform, artificial intelligence, social innovation

Mots-clés : collaboration, plateforme, intelligence artificielle, social innovation

\section{AUTEUR}

\section{DAMIEN RENARD}

Institut Langage \& Communication, Université catholique de Louvain 